\title{
Editorial
}

\section{Vascular Catheters Inserted in the Trenches Versus Guideline Documents: Can the Discrepancies Be Resolved?}

\author{
Robert J. Sherertz, MD; William R. Jarvis, MD
}

Infection control personnel familiar with the studies underlying recent guideline recommendations for the prevention of intravascular catheter-related infections ${ }^{1}$ could conclude that it would be easy to minimize the risk of such infections. Randomized, controlled trials have demonstrated that prepping the skin with chlorhexidine antiseptic can reduce the risk of catheter-related bloodstream infection (BSI) to less than $1 \%,{ }^{2}$ using maximal sterile barriers also can reduce the risk of catheter-related BSI to less than $1 \%,{ }^{3}$ choosing a central venous catheter (CVC) with anti-infective properties can reduce the risk of catheter-related BSI to $1 \%$ or less, ${ }^{4-6}$ and, finally, education can reduce the risk of catheter-related BSI associated with physicians-in-training. ${ }^{7,8}$ Yet, despite these impressive results in short prospective studies, in practice it is uncommon to find endemic catheter-related BSI rates less than $1 \%$.

In this issue of Infection Control and Hospital Epidemiology, there are two large prospective observational studies that address the in-hospital standard of practice related to $\mathrm{CVCs}^{9,10}$ Alonso-Echanove et al. performed a study sponsored by the Centers for Disease Control and Prevention (CDC) of 4,535 patients with CVCs in 8 intensive care units (ICUs) in 6 different National Nosocomial Infections Surveillance (NNIS) System hospitals in the United States between 1997 and 1999 that examined 60 potential risk factors for catheter-related BSI. ${ }^{9}$ Braun et al. performed a study sponsored by the Society for Healthcare Epidemiology of America (SHEA), the Joint Commission on Accreditation of Healthcare Organizations (JCAHO), and the $\mathrm{CDC}$ regarding the standard of practice associated with 3,320 CVC insertions in ICU patients in 55 hospitals (41 in the United States and 14 from other countries) between 1998 and $1999 .^{10}$ The large size of both of these studies gave them substantial power to examine questions previously not answerable at single institutions. Several important observations were made.

Braun et al. found that $91 \%$ of the CVCs inserted were nontunneled, $85 \%$ had multiple lumens, and $22 \%$ were impregnated with antimicrobials. ${ }^{10}$ Forty-four percent of the CVCs were inserted in the internal jugular vein, followed by $32 \%$ in the subclavian location. Fifty-eight percent of the time, a large drape was used at the time of CVC insertion. Although the clinicians inserting the CVCs were experienced (median of $30 \mathrm{CVC}$ insertions in the past 6 months), $12 \%$ of the time insertions were attempted at two or more sites and $20 \%$ of the time two or more attempts were required at the final insertion site. Overall, few CVCs $(<20 \%)$ were managed by an intravenous therapy team as opposed to ICU nurses, despite the fact that intravenous teams have been associated with lower catheter-related BSI rates. More than $50 \%$ of the time nurse staffing involved float nurses and more than $30 \%$ of the time nurse staffing involved agency staff, although float nurses made up only $8 \%$ of the 15 nurse-to-patient-days ratio that was the average during the study. Both a lower nurse-to-patient ratio and the use of float nurses have been associated with higher catheter-related BSI rates in ICU patients. ${ }^{11-13}$ The catheter-related BSI rates for the participating institutions were not provided in this publication, but it is hoped that an additional publication will use multivariate analysis to determine which of the practices they examined have the greatest independent effect on the risk of catheter-related BSI.

Alonso-Echanove et al. found that the average duration of CVC placement was 6.6 days; CVCs with multiple lumens made up $88 \%$ of the CVCs; CVCs were most commonly inserted in the internal jugular vein (44\%), followed by the subclavian vein $(37 \%) ; 21 \%$ of the CVCs were impreg- 
nated with antimicrobials; and the overall catheter-related BSI rate was $2.8 \% .{ }^{9}$ Of 60 potential risk factors for catheterrelated BSI, 27 were significantly associated with the risk of infection, but only 6 were independent risk factors for catheter-related BSI by Cox proportional hazard modeling: (1) patient cared for by float nurse-days more than $60 \%$ of the time (hazard ratio, 2.75); (2) receipt of hyperalimentation through a nonimpregnated CVC (hazard ratio, 2.2); (3) no antimicrobials for the 48 hours after CVC insertion (hazard ratio, 1.94); (4) patient unarousable for more than $70 \%$ of the time (hazard ratio, 1.8); (5) patient age from 45 to 55 years (hazard ratio, 1.57); and (6) the use of a peripherally inserted central catheter (PICC) (hazard ratio, 0.23).

From the perspective of guidelines, these two studies raise several interesting points. First, currently it is strongly felt that the subclavian vein is the optimal site for CVC placement (category IA), ${ }^{1}$ yet both studies found that the internal jugular vein was most frequently used. The explanation for this was not provided in either study, but in the study by Braun et al. (20\%) ${ }^{10}$ and in most ICUs, a large proportion of ICU patients have their CVCs inserted in the operating room by anesthesiologists, who anecdotally prefer the internal jugular route of CVC insertion. Second, only approximately $20 \%$ of the CVCs inserted had an anti-infective coating in both studies and more than $95 \%$ of the impregnated CVCs were coated with silver sulfadiazine plus chlorhexidine. Although it is a category IA recommendation that antiseptic-impregnated CVCs should be considered in ICU patients, it is unclear what the optimal use of coated catheters is in the ICU setting. For example, shouldn't all efforts be made to ensure that the current intravenous guideline recommendations are actual practice in the ICU (ie, use of chlorhexidine skin antiseptic for CVC preparation and use of maximal barriers during CVC insertion) and that elevated catheter-related BSI rates persist before antiseptic-impregnated CVCs are introduced? The study by Alonso-Echanove et al. even raises the possibility that only patients receiving hyperalimentation may benefit from antiseptic-impregnated $\mathrm{CVCs} .{ }^{9}$ Also of interest is the finding that the minocycline and rifampin-coated catheter, which a 1999 study found was superior to the chlorhexidine and silver sulfadiazine-coated catheter, ${ }^{6}$ was only in limited use. Although this may only represent the lack of awareness of this catheter at the time of the study, it could also represent concerns that have been raised about antibiotic resistance, which one recent study suggested may be a real concern. ${ }^{14}$ Third, Braun et al. found that large sterile drapes were not used $41 \%$ of the time. ${ }^{10}$ Given that the benefits of using maximal sterile barriers have been known for approximately 10 years ${ }^{3}$ and recent guidelines have made this a category IA recommendation, ${ }^{1}$ these data suggest that knowledge has either not penetrated the CVC inserter population or not been accepted by these clinicians. All three of these findings provide evidence of the range of difficulties associated with translating guideline recommendations into clinical practice and emphasize that continuing efforts need to be made to identify and eliminate barriers to adopting improvements and changing physician practices. ${ }^{15-17}$

Several additional findings from the study by Alonso-
Echanove et al. raise questions that suggest further studies of catheter-related BSI and CVC insertion and manipulation practices need to be done. ${ }^{9}$ The finding that no antibiotics for the first 48 hours after CVC insertion increased the risk of catheter-related BSI raises the question of whether prophylactic antimicrobials for CVC insertion may be of value. Four of five randomized trials examining this question in oncology patients have suggested that antimicrobials can reduce the risk of catheter-related BSI. ${ }^{18-22} \mathrm{~A}$ similar study should be performed on ICU patients to see whether the same thing is true in that population. The use of PICCs had a $75 \%$ lower risk of catheter-related BSI than did the use of other CVCs. Is this truly an indication that PICCs have a lower risk of catheter-related BSI, or is it just a marker that patients with PICCs were less severely ill? In one small randomized trial comparing PICCs with subclavian CVCs for hyperalimentation in hospitalized patients, the rates of catheter-related BSI were similar in the two groups and the PICC group had a higher rate of thrombophlebitis. ${ }^{23}$ Further study will be necessary to determine whether PICCs are associated with a lower risk of catheter-related BSI in ICU patients. Finally, one of the most interesting findings was that float nurses were associated with a higher risk of catheter-related BSI. A great deal of concern has been raised in recent years about the impact of reduced nurse staffing on patient outcomes. Several studies have shown that a decrease in the ratio of nursing staff to patients can lead to increased morbidity, including an increased risk of catheter-related BSI, ${ }^{11,13,24,25}$ and to increased mortality. ${ }^{26-29}$ The findings of Alonso-Echanove et al. extend this concern further by demonstrating that having an increased proportion of nurses with less than 1 year of experience in the ICU can lead to an increased risk of catheter-related BSI. Thus, pulling nurses from other units or from nursing agencies just to maintain a certain ratio of nurses to patients may not be a satisfactory solution. This increases the difficulties associated with nursing shortages and declining hospital reimbursement. It also means that when increased catheter-related BSI rates are being investigated, this variable will have to be included as part of such investigations.

The two studies do have limitations. Neither the hospitals nor the patients were selected randomly, leaving open the possibility of selection bias. There was no validation of the methodology and, in particular, no systematic training of the data gatherers. Although the methods of the study sponsored by SHEA, JCAHO, and the CDC are not provided, the initial protocol indicated that the numerator and denominator data did not need to be collected simultaneously, but rather that denominator data collected during one period could be used with numerator data collected during another period. No analysis was done controlling for severity of illness, multiple data gatherers, or other factors. Despite these limitations, the size of the study suggests that the findings may be significant and warrant further investigation.

Finally, it is important to point out that the findings of the two studies under consideration could not have been 
accomplished without the large, multicenter design of both of the studies. In particular, the CDC played an important role in both of the studies with NNIS System hospitals making up either all (ie, the study by Alonso-Echanove et al.) or the largest proportion (ie, the study by Braun et al.) of the hospitals in each study. In recent years, the reassignment of NNIS System personnel within the Division of Healthcare Quality Promotion of the CDC to other priority areas (including bioterrorism preparedness and the severe acute respiratory syndrome), and the discontinuation of the Intensive Care Antibiotic Resistance Epidemiology (ICARE) study (an important collaboration among the $\mathrm{CDC}$, the pharmaceutical industry, and academia) at a subset of NNIS System hospitals, has seriously impaired the ability of the Division of Healthcare Quality Promotion personnel to maintain the NNIS System. The NNIS System has contributed benchmark data for hospitals in the United States and throughout the world. It is critical that, during this time of emphasis on patient safety, emerging infections, and bioterrorism preparedness, sufficient support be provided to maintain the NNIS System, which has been a model surveillance system for nosocomial infections. Without the selfless, voluntary participation of the NNIS System hospitals in these studies, it is highly unlikely such studies would have been conducted. Until extramural research funding from the $\mathrm{CDC}$ and the National Institutes of Health is expanded to include nosocomial infection prevention, it is imperative that sufficient support be provided within the CDC to ensure that the NNIS System survives. Nosocomial infections are a major cause of adverse patient outcomes and their prevention is fostered by publication of these NNIS System data.

\section{REFERENCES}

1. O'Grady NP, Alexander M, Dellinger EP, et al. Guidelines for the prevention of intravascular catheter-related infections. Infect Control Hosp Epidemiol 2002;23:759-769.

2. Maki DG, Alvarado CJ, Ringer MA. A prospective, randomized trial of povidone-iodine, alcohol and chlorhexidine for prevention of infection with central venous and arterial catheters, Lancet 1991;338:339-343.

3. Raad II, Hohn DC, Gilbreath BJ, et al. Prevention of central venous catheter-related infections by using maximal sterile barrier precautions during insertion. Infect Control Hosp Epidemiol 1994;15:231-238.

4. Raad I, Darouiche R, Dupuis J, et al. Central venous catheters coated with minocycline and rifampin for the prevention of catheter-related colonization and bloodstream infections: a randomized, double-blind trial. Ann Intern Med 1997;127:267-275.

5. Maki DG, Stolz SM, Wheeler S, Mermel LA. Prevention of central venous catheter-related bloodstream infection by use of an antisepticimpregnated catheter: a randomized, controlled trial. Ann Intern Med 1997;127:257-265.

6. Darouiche RO, Raad II, Heard SO, et al. A comparison of two antimicrobial-impregnated central venous catheters. N Engl J Med 1999;340:1-8.

7. Sherert $z$ RJ, Ely EW, Westbrook DM, et al. Education of training physicians can decrease the risk of vascular catheter infections. Ann Intern Med 2000;132:641-648.

8. Eggimann $\mathrm{P}$, Harbarth $\mathrm{S}$, Constantin $\mathrm{MN}$, et al. Impact of a prevention strategy targeted at vascular-access care on incidence of infections acquired in intensive care. Lancet 2000;355:1864-1868.
9. Alonso-Echanove J, Edwards JR, Richards MJ, et al. Effect of nurse staffing and antimicrobial-impregnated central venous catheters on the risk for bloodstream infections in intensive care units. Infect Control Hosp Epidemiol 2003;24:916-925.

10. Braun BI, Kritchevsky SB, Wong ES, et al. Preventing central venous catheter-associated primary bloodstream infections: characteristics of practices among hospitals participating in the Evaluation of Processes and Indicators in Infection Control (EPIC) study. Infect Control Hosp Epidemiol 2003;24:926-935.

11. Fridkin SK, Pear SM, Williamson TH, Galgiani JN, Jarvis WR. The role of understaffing in central venous catheter-associated bloodstream infections. Infect Control Hosp Epidemiol 1996;17:150-158.

12. Archibald LK, Ramos M, Arduino MJ, et al. Enterobacter cloacae and Pseudomonas aeruginosa polymicrobial bloodstream infections traced to extrinsic contamination of a dextrose multidose vial. I Pediatr 1998;133:640-644.

13. Robert J, Fridkin SK, Blumberg HM, et al. The influence of the composition of the nursing staff on primary bloodstream infection rates in a surgical intensive care unit. Infect Control Hosp Epidemiol 2000;21:12-17.

14. Wright F, Heyland DK, Drover JW, McDonald S, Zoutman D. Antibioticcoated central lines: do they work in the critical care setting? Clinical Intensive Care 2001;12:21-28.

15. McNeil BJ. Shattuck lecture: hidden barriers to improvement in the quality of care. $N$ Engl J Med 2001;345:1612-1620.

16. Greco PJ, Eisenberg JM. Changing physician practices. $N$ Engl J Med 1993;329:1271-1274.

17. Cabana MD, Rand CS, Powe NR, et al. Why don't physicians follow clinical practice guidelines? A framework for improvement. JAMA 1999;282:1458-1465.

18. Harms $\mathrm{K}$, Herting $\mathrm{E}$, Kron $\mathrm{M}$, et al. Randomized, controlled trial of amoxicillin prophylaxis for prevention of catheter-related infections in newborn infants with central venous silicone elastomer catheters. $J$ Pediatr 1995;127:615-619.

19. Vassilomanolakis M, Plataniotis G, Koumakis G, et al. Central venous catheter-related infections after bone marrow transplantation in patients with malignancies: a prospective study with short-course vancomycin prophylaxis. Bone Marrow Transplant 1995;15:77-80.

20. Lim SH, Smith MP, Machin SJ, Goldstone AH. A prospective randomized study of prophylactic teicoplanin to prevent early Hickman catheterrelated sepsis in patients receiving intensive chemotherapy for haematological malignancies. Eur J Haematol Suppl 1993;54:10-13.

21. Bock SN, Lee RE, Fisher B, et al. A prospective randomized trial evaluating prophylactic antibiotics to prevent triple-lumen catheter-related sepsis in patients treated with immunotherapy. J Clin Oncol 1990;8:161169.

22. McKee R, Dunsmuir R, Whitby M, Garden OJ. Does antibiotic prophylaxis at the time of catheter insertion reduce the incidence of catheterrelated sepsis in intravenous nutrition? J Hosp Infect 1985;6:419-425.

23. Cowl CT, Weinstock JV, Al-Jurf A, Ephgrave K, Murray JA, Dillon K. Complications and cost associated with parenteral nutrition delivered to hospitalized patients through either subclavian or peripherally-inserted central catheters. Clin Nutr 2000;19:237-243.

24. Haley RW, Bregman DA. The role of understaffing and overcrowding in recurrent outbreaks of staphylococcal infection in a neonatal specialcare unit. I Infect Dis 1982;145:875-885.

25. Kovner C, Gergen PJ. Nurse staffing levels and adverse events following surgery in U.S. hospitals. Image Journal of Nursing Schools 1998;30:315321.

26. Sasichay-Akkadechanunt T, Sealzi CC, Jawad AF. The relationship between nurse staffing and patient outcomes. Journal of Nursing Administration 2003;33:478-485.

27. Needleman J, Buerhaus P, Mattke S, Stewart M, Zelevinsky K. Nursestaffing levels and the quality of care in hospitals. $N$ Engl J Med 2002;346:1715-1722.

28. Aiken LH, Clarke SP, Sloane DM, Sochalski J, Silber JH. Hospital nurse staffing and patient mortality, nurse burnout, and job dissatisfaction. JAMA 2002;288:1987-1993.

29. Tucker J, UK Neonatal Staffing Study Group. Patient volume, staffing, and workload in relation to risk-adjusted outcomes in a random stratified sample of UK neonatal intensive care units: a prospective evaluation. Lancet 2002;359:95-96. 